\title{
Wie hältst Du es mit der Konkurrenz?
}

\section{Der Vertragswettbewerb zwischen Feinsteuerung und grundlegendem Reformbedarf}

\section{STEPHAN FELDMANN}

Stephan Feldmann ist Referent in der Abteilung Ambulante Versorgung beim AOKBundesverband in Berlin

In ihrem Diskussionsbeitrag problematisieren Dominik von Stillfried und Thomas Czihal das Nebeneinander von Kollektiv- und Selektivverträgen, das sie durch mangelnde Datentransparenz und methodische Unschärfen in der Weiterentwicklung der vertragsärztlichen Vergütung beeinträchtigt sehen. Tatsächlich bedarf der Vertragswettbewerb im Gesundheitswesen einer umfassenden Bestandsaufnahme. Die von vielen erhofften und von anderen befürchteten Umwälzungen in der Versorgungslandschaft sind weitgehend ausgeblieben und die Prognose deutet auf mittlere Sicht bei einem unveränderten Ordnungsrahmen eher auf Stillstand hin. Die Ursachen hierfür liegen in grundlegenden Regelungsdefiziten, die wesentliche Parameter des Vertragswettbewerbs offen lassen. Die legislativen Versäumnisse der vergangenen Legislaturperioden haben verhindert, dass ein für die Akteure verlässlicher und diskriminierungsfreier Wettbewerbsrahmen entstehen konnte.

\section{Einleitung}

Die Weiterentwicklung des Wettbewerbs im Gesundheitswesen ist in der politischen Rahmensetzung zunehmend in den Hintergrund getreten. Der Gesetzgeber bot im zurück liegendem Versorgungsstrukturgesetz in dieser Frage keine nennenswerten Impulse, sondern entschied sich vielmehr in den Regelungen zur ambulanten spezialfachärztlichen Versorgung aktiv gegen wettbewerbliche Elemente. Solche waren zwar in der Diskussion, aber derzeit ist regelmäßig zu beobachten, dass Wettbewerb zwar von verschiedenen Seiten gefordert wird, aber die Türen zur legislativen Umsetzung bemerkenswerterweise zu Zeiten eines liberal geführten Gesundheitsministeriums - weitgehend verschlossen blieben.

Öffneten sich die Türen, erschöpfetn sich die Debatten um den Vertragswettbewerb in der immer wieder aufflammenden Streitfrage, ob für die Hausarztverträge nach $\$ 73 \mathrm{~b}$ SGB V das Refinanzierungsgebot weiter gelten oder aufgehoben werden sollte. Diese Kontroverse, die insbesondere aufgrund des Engagement Bayerns sogar den Bundesrat beschäftigt hat, verstellt in ihrer 
Verengung auf die monetären Aspekte der Selektivverträge den Blick auf die übrigen, für einen funktionierenden Vertragswettbewerb nachhaltigen Lösungsansätze.

Daher ist es durchaus verdienstvoll, wenn sich Stillfried und Czihal in ihrem Debattenbeitrag dieser Frage zuwenden. Denn tatsächlich ist die Suche nach einer richtigen Balance zwischen kollektivem Sicherstellungsinteresse und dem angemessenen und erforderlichen $\mathrm{Maß}$ an selektiver Vertragsfreiheit zur Steigerung von Qualität und Effizienz im Gesundheitswesen bei weitem noch nicht abgeschlossen. Noch immer ist ein fairer und diskriminierungsfreier Wettbewerb zwischen Kollektiv- und Selektivverträgen, zwischen KVen und konkurrierenden Leistungsanbietern nicht hergestellt. In diesem Beitrag sollen aus einem anderen Blickwinkel diejenigen offenen Regelungskreise diskutiert werden, deren Unvollkommenheit bislang einen wirksamen Vertragswettbewerb im ambulanten Bereich hemmen. Im Anschluss soll der Ansatz von Stillfried und Czihal kommentiert werden.

\section{Der Vertragswettbewerb}

Der Wettbewerb in der ambulanten Versorgung ist durch das Zusammenspiel von Kassen- und Vertragswettbewerb gekennzeichnet. Durch die Einführung der freien Kassenwahl für Versicherte und dem (mittlerweile morbiditätsorientierten) Risikostrukturausgleich wurde bereits in den 90er Jahre der Wettbewerb zwischen den Krankenkassen begründet. Sie sollten durch die Konkurrenz um Versicherte ihre Leistungsfähigkeit und Wirtschaftlichkeit sowie das Versorgungsangebot verbessern. Ein auf Service und Satzungsleistungen reduzierter Wettbewerb kann diesen Anspruch allerdings nicht einlösen, wenn er nicht mit umfänglichen Freiheiten zur Gestaltung der Versorgung einhergeht.

Anderseits liefe der Vertragswettbewerb leer, wenn nicht gleichzeitig die Kassen ihrerseits im Wettbewerb um Versicherte stehen und der Unternehmenserfolg eine Funktion der Vertragspolitik ist. Vertragswettbewerb und Kassenwettbewerb sind daher zwei Seiten ein und derselben Medaille, die zusammenwirken und sich gegenseitig bedingen. Durch den Abschluss von Ver- sorgungsverträgen wird Krankenkassen ermöglicht, hinsichtlich Preis und Qualität ein besseres Angebot zu unterbreiten, womit sie komparative Vorteile gegenüber anderen Krankenkassen im ständigen Werben um Versicherte erzielen können. Gleichzeitig wird durch das Recht der Krankenkassen auf Auswahl ihrer Vertragspartner der Wettbewerb zwischen den Leistungserbringern gefördert.

Die Funktion des Vertragswettbewerbs geht somit über die Leuchtturmhypothese, die auch von Stillfried und Czihal angeführt wird, deutlich hinaus, da in dieser Sichtweise die Interaktion

\section{Noch immer ist ein fairer und diskriminierungsfreier Wettbewerb zwischen Kollektiv- und Selektivvertrag nicht hergestellt.}

mit dem Wettbewerb auf Krankenkassenebene nicht hinreichend berücksichtigt wird. Dies kann auch zu irrigen Vorstellungen zur vermeintlichen Weiterentwicklung des Wettbewerbs verleiten, wie die am 01.03.2013 verabschiedete Position der KBV zum Vertragswettbewerb belegt. Darin wird die regelhafte und finanzneutrale Überführung von selektivvertraglichen Versorgungsverbesserungen und Innovationen in die Regelversorgung gefordert.

Diese Kollektivierung selektivvertraglicher Errungenschaften wird rhetorisch als Patienteninteresse vermarktet, stellt in erster Linie aber eine Innovationsbremse zum Nachteil der Versorgung dar. Denn sie enthebt die Wettbewerber sowohl auf Kassen- als auch auf Leistungserbringerebene von der Verantwortung, eigene Anstrengung zur Nachahmung zu unternehmen. Dies betrifft insbesondere die Kassenärztlichen Vereinigungen selbst, die sich gewissermaßen zum Nulltarif Innovationen aus selektiven Versorgungsverträgen sichern möchten. Der Anreiz für Krankenkassen, in neue Versorgungsformen zu investieren, wird dadurch deutlich reduziert, sodass die Innovationsfähigkeit erlahmt. Der behauptete Patientennutzen ist hingegen nur eine Schimäre, da der Zugang zu Versorgungsverbesserungen durch die weitreichenden Möglichkeiten des Kassenwechsels bereits gewährleistet ist. Diese Perspektive der Versichertenwanderung verstärkt gleichzeitig den Druck auf Wettbewerber, ihrerseits in Innovationen zu investieren.

\section{Hemmnisse des Vertragswettbewerbs}

Trotz der seit mehreren Jahren bestehenden Möglichkeiten haben die Krankenkassen von ihrer Option, Verträge ohne Beteiligung der KVen zu schließen, nur sehr zurückhaltend Gebrauch gemacht. Mehrheitlich suchen die Kassen vertragliche Lösungen mit den KVen (oder mit deren Beteiligung) in Form von sogenannten Add-on-Verträgen, die lediglich versorgungsergänzend und nicht versorgungsersetzend wirken. Da gleichzeitig aber die Anreize des Morbiditätsorientierten Risikostrukturausgleichs wirken, erscheint diese Vertragsstrategie nicht rational. Wie im folgenden gezeigt wird, existieren aber allen gesetzlichen Initiativen zum Trotz weiterhin wettbewerbshemmende Faktoren, die eine von den kollektiven Strukturen losgelöste Vertragspolitik erschweren.

\section{Rechtsunsicherheit im Zusammenhang mit dem Sicherstellungsauftrag}

Im Gesetz sind Art und Umfang des Übergangs und der Ausübung des Sicherstellungsauftrages auf die Krankenkassen nur unzureichend geregelt. In welchem Maße sich der Gesetzgeber in diesem Punkt einer klaren Abgrenzung der Verantwortlichkeit zwischen KVen und vertragschließender Krankenkasse enthalten hat, wird bereits daran deutlich, dass es sogar an einer klaren Zuweisung an die Krankenkassen mangelt. Das Gesetz ordnet seinem Wortlaut nach im Falle eines Selektivvertrags lediglich die Einschränkung des Sicherstellungsauftrags für die Kassenärztlichen Vereinigungen an. Damit ist selbst die grundlegende Frage, wer anstelle der Kassenärztlichen Vereinigungen die Versorgung gewährleisten soll, nur im Wege der Rechtsauslegung zu ermitteln. Ein gesetzlicher Rahmen zu den Instrumenten, die den Krankenkassen zur Sicherstellung zur Verfügung stehen oder in welches Verhältnis die voneinander getrennten Sicherstellungsaufträge 
zueinander gesetzt werden sollen, fehlt vollständig. Dieses Regelungsvakuum kann zu erheblichen Spannungen führen, wenn die KVen mit Hinweis auf die selektivvertraglich versorgten Versicherten die gemeinsame Wahrnehmung von Aufgaben der Sicherstellung, dazu gehören die Bedarfsplanung, die Qualitätssicherung oder die Wirtschaftlichkeitsprüfung in Frage stellen. In diesen Zusammenhang ist auch der Hinweis von Stillfried/Czihal auf die reduzierte Datenlage in der Bedarfsplanung zu stellen.

Derartige Unsicherheiten stellen für die Krankenkassen zusätzliche Transaktionskosten dar, da mögliche Konflikte mit den Kassenärztlichen Vereinigungen bewertet und in das wirtschaftliche Kalkül des Selektivvertrages miteinbezogen werden müssen.

Dieser von Gesetzes wegen anarchische Dualismus eines geteilten Sicherstellungsauftrages kann allerdings nicht dadurch behoben werden, dass mehr Datensätze hin- und hergeschoben werden, wie es Stillfried/Czihal nahelegen. Dies wäre eine Verkennung des grundlegenden legislativen Handlungsbedarfs zur Formulierung eines trennscharfen Sicherstellungsauftrages mit einem gesetzlich normierten Instrumentarium, dass den Kassen ermöglicht, ihre erworbene Verpflichtung auch rechtssicher wahrzunehmen.

\section{Die Bereinigung der Kollektivbudgets}

Mehr Fortschritte sind in der Frage der Bereinigung der Budgets zu beobachten, gleichwohl sind hier nur in Teilen befriedigende Lösungen vorhanden. Die sachgerechte Bereinigung der Kollektivbudgets stellt als Maßnahme zur Verhinderung der Doppelfinanzierung eine wesentliche, vielleicht sogar die bedeutsamste Voraussetzung für Selektivverträge ohne $\mathrm{KV}$-Beteiligung dar. Hier hat die gesetzliche Kompetenzzuweisung an den Bewertungsausschuss, hierzu Vorgaben zu erlassen, durchaus für ein höheres Maß an Klarheit gesorgt.

Bemerkenswert bleiben allerdings die erheblichen Mitwirkungsrechte der KBV und der KVen. Es kommt im deutschen Wirtschaftsleben nicht häufig vor, dass einem Mitbewerber der unmittelbare Einfluss auf die finanziellen Rahmenbedingungen der Konkurrenz eingeräumt wird. Durch die Mitwirkung zum Abschluss einer Vereinbarung erhalten die KVen vielfältige Möglichkeiten, die Bereinigung zu erschweren oder zu verzögern, während die Krankenkassen gleichzeitig zum Abschluss von Hausarztverträgen durch Schiedsamtsbeschluss gezwungen werden können. Diese asymmetrischen Verhandlungspositionen sowie die Option, die Bereinigungsvereinbarung seinerseits durch ein Schiedsamt festsetzen zu lassen, fördert Kompromisslösungen, die nicht immer sach- und aufwandsgerechte Bereinigungen ermöglichen.

\section{Das grundlegende Wettbewerbshemmnis ist die marktbeherrschende Stellung der KVen.}

Noch kritischer ist der Bedarf nach sachgerechten Regelungen bei der Bereinigung arzt- bzw. arztgruppenbezogener Teilbudgets. Seit dem Versorgungsstrukturgesetz haben die KVen hier die alleinige Gestaltungsautonomie, die sie in diesem Zusammenhang dazu nutzen, die alleinige Last der Bereinigung den an Selektivverträgen teilnehmenden Ärzten aufzubürden, auch wenn dies durch die geltenden Honorarverteilungsregeln (z.B. bei der Berechnung von Regelleistungsvolumina anhand $\mathrm{KV}$-durchschnittlicher Fallwerte) sachlich nicht gerechtfertigt ist. Die daraus resultierende überproportionale Belastung der teilnehmenden Ärzte mündet in Forderungen an die vertragsschließende Kasse, die zusätzlichen Honorarausfälle durch höhere Vergütungen im Selektivvertrag zu kompensieren.

\section{Marktmacht der KVen}

Die neu entstandene Problematik der arztseitigen Bereinigung leitet zu einem grundsätzlichen Hemmnis des Vertragswettbewerbs über - der marktbeherrschenden Stellung der Kassenärztlichen Vereinigung. Diese äußert sich nicht nur in ihren Mitsprache- und Behinderungsmöglichkeiten bei der Bereinigung, sondern insbesondere in der durch das Monopol im Kollektivvertrag aufrechterhaltenen und bereits finanzierten Infrastruktur zum Management von Verträgen. Aus diesem Grund haben die KVen im Wettbewerb um Selektivver- träge unbestreitbare Vorteile gegenüber Mitbewerbern, die diese Strukturen erst aus eigener Kraft aufbauen müssen. Selbst wenn dies gelingt, bleiben diese Parallelstrukturen für viele Ärzte unattraktiv, da zusätzlich neue Prozesse neben den etablierten Abrechnungsweg der KV aufzusetzen sind.

Inwieweit die marktbeherrschende Stellung der KVen mit den Mitteln des Kartellrechts, insbesondere der Missbrauchsaufsicht, neutralisiert werden könnte, ist zweifelhaft. Es könnte vielmehr geboten sein, aufgrund der spezifischen Umstände in der vertragsärztlichen Versorgung Teilaufgaben aus dem Organisationsbereich der KVen herauszulösen und in von Dritter Seite verwaltete Zweckgemeinschaften auszulagern, sodass auch Wettbewerber der KVen diese Infrastruktur nutzen können. Ein denkbares Beispiel wäre die Abwicklung des Datenträgeraustausches zur Abrechnung zwischen den Ärzten und den KVen.

\section{Auswirkungen der Vertragshemmnisse auf die Vertragspolitik der Kassen}

Den vorgenannten Vertragshemmnissen ist gemein, dass sie die Transaktionskosten der Selektivverträge systematisch erhöhen und die Marktposition der KVen weiter stärken. Zudem existieren hinreichend Anreize und Ansatzpunkte für die KVen mit einer Konfrontationsstrategie diese Wirkungen zu steigern und die Belastung für die Krankenkassen zu erhöhen.

Aus Sicht der Krankenkasse sind Selektivverträge daher mit einer Vielzahl von Unwägbarkeiten verbunden, die sie von einer aktiven Vertragspolitik ohne Beteiligung der Kassenärztlichen Vereinigungen abhalten können. Es bedarf einigen Unternehmergeistes und versorgungspolitischen Anspruchs, aber auch die Bereitschaft Konflikte mit den Kassenärztlichen Vereinigungen zu führen und durchzuhalten, um trotzdem mithilfe von Direktverträgen Innovationen in die ambulante ärztliche Versorgung einzuführen.

\section{Der Ansatz von Stillfried/Czihal}

Dem vorstehend gezeichneten Gebirgspanorama des angehäuften Handlungsbedarfs, für das noch nicht einmal An- 
spruch der Vollständigkeit erhoben werden kann, steht die ausgeprägt technisch orientierte Problemskizze von Stillfried/ Czihal gegenüber. Die Autoren beschränken sich in ihrer Analyse auf einen relativ speziellen Bereich der vertragsärztlichen

\section{Methodischen Detailfragen wird ein Rang eingeräumt, der ihnen tatsächlich nicht zusteht.}

bereits auf Referenten- oder Abteilungsleiterebene - denn nur dort werden die Modelle wirklich verstanden - um jedes Detail erbittert gerungen. Diese neu gewonnene Bedeutung verleitet dazu, solchen methodischen Einzelfragen - wie der sachgerechten Berücksichtigung von Selektivvertragsteilnehmern - auch im politischen Diskurs einen Rang einzuräumen, den ihnen bei Licht besehen im Regelungsgefüge der ambulanten

Vergütung, der Bestimmung der morbiditätsbedingten, genauer diagnosebezogenen Veränderungsrate.

Die von den Autoren diskutierte Frage lautet im Kern, ob und inwieweit die Teilnehmer an bereinigten Selektivverträgen im Rahmen der noch in Anspruch genommenen Kollektivversorgung eine andere Entwicklung der diagnosebezogenen Morbidität aufweisen als die Restpopulation. Dies wäre beispielsweise der Fall, wenn bei den Teilnehmern an einem bereinigten Hausarztvertrag hinsichtlich der von Fachärzten gestellten Diagnosen eine höhere (aber auch niedrigere) Prävalenzentwicklung beobachten werden kann als bei Versicherten, die ausschließlich kollektivvertraglich versorgt werden.

Damit wird ein Anliegen der Kassenärztlichen Vereinigungen, insbesondere aus dem Süden der Republik adressiert, dass sowohl im vergangenen als auch in diesem Jahr Gegenstand kontroverser Diskussionen im Bewertungsausschuss gewesen ist.

\section{Das Primat des Technischen}

Es bedarf schon mehr als einer gewissen Liebe zum Detail, dieser rein methodischen Frage der Modellgestaltung eine existenzielle Bedeutung für das Verhältnis von Kollektiv- und Selektivvertrag beizumessen. Gleichwohl ist diese Fokussierung auf die methodische Feinsteuerung Ausdruck der zunehmenden Delegation politischer Entscheidungen an statistische Expertensysteme, wie im vorliegenden Beispiel das Klassifikationsmodell zur Ermittlung der morbiditätsbedingten Veränderungsrate. $\mathrm{Da}$ einzelne Modellentscheidungen Auswirkungen auf die Ergebnisse haben können, die noch vor wenigen Jahren langwierige Verhandlungen auf höchster Ebene benötigt hätten, wird inzwischen
Versorgung nicht zukommt.

Zudem tritt eine Machbarkeitsillusion in Erscheinung, die den Ergebnissen derartiger Berechnungsmodelle den Rang einer objektiven Wahrheit beimessen. Bereits der Gebrauch des Begriffs der „Fehlschätzung“ oder „falsch-niedrige Schätzung “ suggeriert eine irrtümliche Abweichung von einem tatsächlichen „wahren“ Wert, der ansonsten durch das Modell bestimmt würde, wären da nicht angeblich diese ärgerlichen Fehler.

Dabei hat jede Schätzung zur morbiditätsbedingten Veränderung so viel mit der Wirklichkeit zu tun, wie die Schattenbilder an Platons Höhlenwand. Die Ergebnisse solcher Modelle finden ihre Legitimation daher auch nicht in ihrer objektiven Aussagekraft, sondern durch politischen Konsens. Denn das richtige Modell gibt es nicht. Vielmehr prägen auch Zweckmäßigkeitserwägungen, empirisch nicht immer nachprüfbare Annahmen und wirtschaftliche Restriktionen die Modellgestaltung. Eine Änderung des Modells zieht unweigerlich auch ein anders Ergebnis nach sich. Die morbiditätsbedingte Veränderungsrate wird nicht deswegen vereinbart, weil sie die richtige wäre, sondern als Ergebnis eines Kompromisses zwischen widerstreitenden Interessen.

Der geradezu heilige Ernst, mit dem der Morbiditätsorientierung dennoch in der vertragsärztlichen Vergütung gehuldigt wird, liegt hingegen weniger in einer fachlichen, sondern wiederum in einer politischen Rationalität begründet. Durch die Scheinobjektivität der Morbiditätsbindung soll die vertragsärztliche Vergütung befriedet werden, indem deren Weiterentwicklung an ein vermeintlich medizinisches Kriterium gebunden wird und nicht länger der ökonomisch orientierten Beschränkung der Grundlohnsummenveränderung unterworfen sein soll. Wie die Entwicklung der ärztlichen Honorare der letzten Jahre zeigt, hat sich dieser Umstieg auch wirtschaftlich positiv für die Vertragsärzte niedergeschlagen.

\section{Unangemessenes Verhältnis von Problem und Lösung}

Die unangemessene Einordnung des von Stillfried/Czihal beschriebenen Problems schlägt sich auch in den Lösungsvorschlägen nieder. In jedem Fall weisen die aufgezeigten Wege in ihrer Tragweite eine im Vergleich mit dem zu behebenden Problem eine deutlich überschießende Tendenz auf. Zudem widersprechen sie jeweils geltendem Recht.

Weder die vollständige Bereinigung von Selektivvertragsteilnehmern noch die Berücksichtigung von Selektivvertragsdiagnosen bei der Ermittlung der diagnosebezogenen Veränderungsrate sind juristisch nach geltender Rechtslage umsetzbar.

Die Vermischung der Bereinigung mit der Ermittlung der Veränderungsrate widerspricht dem exklusiven Ziel der Bereinigung zur Vermeidung einer Doppelfinanzierung. Stattdessen bürdete diese Lösung den Krankenkassen neue finanzielle Risiken auf, die ihnen durch die nunmehr vollständige extrabudgetäre Vergütung aller der von

\section{Durch die Scheinobjektivität der Morbiditätsbindung soll die vertragsärztliche Vergütung befriedet werden.}

Teilnehmern an Selektivverträgen in Anspruch genommenen Leistungen entstünden. Dies hat der Gesetzgeber von vorneherein unterbinden wollen, indem er den Umfang der Bereinigung ausdrücklich an den im Selektivvertrag vereinbarten Leistungsinhalt gebunden hat. Umgekehrt gelten für Leistungen, die nicht vom Versorgungsumfang eines Selektivvertrages umfasst sind, weiterhin die gesamtvertraglichen Regelungen.

In rechtlicher wie sachlicher Hinsicht ebenso wenig tragfähig ist der Alternativvorschlag, die in Selektivverträgen dokumentierten Diagnosen (SV-Diagnosen) in die Berechnung der diagno- 
sebezogenen Veränderungsrate miteinzubeziehen. Damit würden die Daten der Selektivvertragsteilnehmer ,vervollständigt", sodass diese nicht weiter aus der Berechnung ausgeschlossen werden müssten. Gleichzeitig würde aber die Entwicklung der diagnosebezogenen Morbidität aus dem Selektivvertrag in die diagnosebezogene Veränderungsrate einfließen, sodass diese in zweifacher Weise finanzwirksam würde: Sowohl über die Vergütung im Selektivvertrag als auch über die Veränderungsrate zur Anpassung des morbiditätsbedingten Behandlungsbedarfs. Die Veränderungen der diagnosebezogenen Morbidität würde die Anpassung der Gesamtvergütung beeinflussen, obwohl die Selektivvertragsteilnehmer gerade im vertraglich definierten Umfang aus der vertragsärztlichen Versorgung herausgenommen werden sollen. In der Logik von Stillfried/Czihal, die von einer überdurchschnittlichen Entwicklung der diagnosebezogenen Morbidität im Selektivertrag ausgehen, würde eine vertragsschließende Krankenkasse einen Morbiditätsanstieg zweimal finanzieren: im Selektivvertrag und in der Kollektivversorgung, wobei in letzterer alle Krankenkassen betroffen wären, also auch solche, die überhaupt keine Selektivverträge haben.

Zudem sind für die Bestimmung der Veränderung der Morbiditätsstruktur lediglich die vertragsärztlichen Diagnosen heranzuziehen $(\mathbb{S} 87 \mathrm{a}$ Abs. 4 Satz 3 SGB V). Ob Diagnosen, die im Rahmen der selektivvertraglichen Versorgung dokumentiert werden, auch als vertragsärztliche Diagnosen zu betrachten sind, ist spätestens mit der jüngsten Vereinbarung zum Bundesmantelvertrag entschieden worden. Dort wird die selektivvertragliche Versorgung explizit außerhalb der vertragsärztlichen Sphäre verortet.

Im Ergebnis entsprechen die Vorschläge von Stillfried/Czihal somit eher der Verhinderungslogik der Kassenärztlichen Vereinigungen, die den Vertragswettbewerb durch eine schrittweise Anhebung der Transaktionskosten des direkten Kontrahierens einzudämmen versuchen, als einem ernsthaften Versuch der Problemlösung. Obgleich die Autoren postulieren, eine „friedliche Koexistenz" der Vertragsformen anzustreben, träte bei einer Umsetzung ihrer Lösungsvorschläge das genaue Gegenteil ein.

\section{Neue Form der Wissenschaftskommunikation:}

\section{Studierende erzählen Geschich- ten und gestalten Bilder}



55 Gründe für mehr Nachhaltigkeit

Ein Projekt von Studierenden der Universität Bremen für eine Welt mit Zukunft

Herausgegeben von Prof. Dr. Georg Müller-Christ

und Dr. Anna Katharina Liebscher

2013, 120 S., brosch., 19,90 €, ISBN 978-3-8487-0945-8

Nachhaltigkeit, etwas genauer betrachtet, kann zuweilen sehr komplex werden. Studierende des Masterstudiengangs Wirtschaftspsychologie an der Universität Bremen haben unter der Leitung von Prof. Dr. Georg Müller-Christ und Dr. Anna Katharina Liebscher aus einer großen Herausforderung 55 kleine Gründe für mehr Nachhaltigkeit gemacht. Alle Gründe zusammen erzählen die Notwendigkeit, mit den materiellen und immateriellen Ressourcen der Welt haushälterisch umzugehen.

Es sind die studentische Sprache und die studentischen Bilder, die in Geschichten, Dialogen und Erläuterungen das Thema Nachhaltigkeit kurzweilig vermitteln. Alle, die in ihrem Alltag mit Nachhaltigkeitsfragen beschäftigt sind, erhalten durch dieses Buch ein umfassenderes Verständnis der großen Herausforderung und erkennen, dass Ökoeffizienz allein die Welt nicht nachhaltiger macht. Wir müssen unsere Ressourcenquellen erhalten und zugleich die Ressourcen effizient einsetzen, lautet die gesamthafte und widersprüchliche Botschaft.

Weitere Informationen unter: www.nomos-shop.de/21946

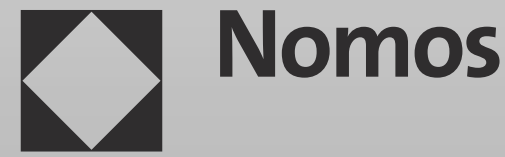

